\title{
Probing the $C P$ structure of the top quark Yukawa coupling: Loop sensitivity versus on-shell sensitivity
}

\author{
Till Martini@ ${ }^{1, *}$ Ren-Qi Pan, ${ }^{2, \dagger}$ Markus Schulze, ${ }^{1, \$}$ and Meng Xiao ${ }^{2, \S}$ \\ ${ }^{1}$ Humboldt-Universität zu Berlin, Institut für Physik, Newtonstraße 15, 12489 Berlin, Germany \\ ${ }^{2}$ Zhejiang Institute of Modern Physics, Department of Physics, Zhejiang University, \\ Hangzhou, Zhejiang 310027, China
}

(Received 13 July 2021; accepted 30 August 2021; published 29 September 2021)

\begin{abstract}
The question whether the Higgs boson is connected to additional $C P$ violation is one of the driving forces behind precision studies at the Large Hadron Collider. In this work, we investigate the $C P$ structure of the top quark Yukawa interaction — one of the most prominent places for searching for New Physicsthrough Higgs boson loops in top quark pair production. We calculate the electroweak corrections including arbitrary $C P$ mixtures at next-to-leading-order in the Standard Model Effective Field Theory. This approach of probing Higgs boson degrees of freedom relies on the large $t \bar{t}$ cross section and the excellent perturbative control. In addition, we consider all direct probes with on-shell Higgs boson production in association with a single top quark or top quark pair. This allows us to contrast loop sensitivity versus on-shell sensitivity in these fundamentally different process dynamics. We find that loop sensitivity in $t \bar{t}$ production and on-shell sensitivity in $t \bar{t} H$ and $t H$ provide complementary handles over a wide range of parameter space.
\end{abstract}

DOI: $10.1103 /$ PhysRevD.104.055045

\section{INTRODUCTION}

The discovery of the Higgs boson by the ATLAS and CMS Collaborations [1-3] in 2012 has sparked an extensive research effort to precisely measure its properties and origin. In fact, it has become one of the main goals of collider phenomenology. Data shows that the discovered particle is consistent with the spin-zero Higgs boson of the Standard Model (SM) [4,5]—within the current uncertainties. One defining property is the $C P$-even Yukawa interaction between the Higgs boson and the SM fermions, which is proportional to the fermion mass. $C P$-violating contributions are in principle allowed by gauge as well as space-time symmetries, and they are theoretically compelling in the context of the baryon asymmetry in the universe. In fact, many generic extensions of the SM, such as twoHiggs-doublet models [6-10] or composite Higgs models [11-25], contain modifications of the simple $C P$ structure of the SM. It is therefore of highest importance to search for

\footnotetext{
till.martini@physik.hu-berlin.de

renqi.pan@cern.ch

${ }^{\ddagger}$ markus.schulze@physik.hu-berlin.de

§meng.xiao@cern.ch
}

Published by the American Physical Society under the terms of the Creative Commons Attribution 4.0 International license. Further distribution of this work must maintain attribution to the author(s) and the published article's title, journal citation, and DOI. Funded by SCOAP ${ }^{3}$.
$C P$-odd contributions that modify the SM interactions. In this regard, the top quark Yukawa interaction stands out due to the large top quark mass, which implies a large coupling. Moreover, top quarks are copiously produced at the LHC, which makes them ideal for searching for New Physics. For example, the CMS and ATLAS experiments [26,27] recently presented first measurements which resulted in the exclusion of a pure $C P$-odd coupling at more than $3 \sigma$, still leaving large room for mixtures of $C P$-even and $C P$ odd components.

In this work, we propose a novel way to probe the $C P$ properties of the top quark Yukawa coupling: We want to challenge the SM at loop level, where the Higgs boson only appears through off-shell degrees of freedom. Hence, we study top quark pair production accounting for electroweak corrections. The leading effects are the $\mathcal{O}(\alpha)$ weak corrections to the QCD-induced $p p \rightarrow t \bar{t}$ process, which we calculate for $C P$-even and $C P$-odd $H t t$ couplings allowing arbitrary mixtures. Our strategy is motivated by the abundant data of top quark pairs produced at the LHC and excellent perturbative control over the theoretical predictions. In fact, the LHC will have produced almost 500 million top quarks by 2023 , while already today, theoretical predictions reach an accuracy of only a few percent [28-33]. These prospects are only getting brighter with the high-luminosity runs commencing in 2027 and high ambitions of the theory community towards next-tonext-to-next-to-leading order $\left(\mathrm{N}^{3} \mathrm{LO}\right)$ calculations [34-36]. Another promising feature is that this approach, using $t \bar{t}$, is 
free from penalties of Higgs boson branching fractions and ambiguities of a complicated final state, which is in contrast to on-shell Higgs production processes such as $p p \rightarrow t \bar{t} H$. Yet, it is at the same order in the perturbative counting.

We note that this idea was actually presented for the first time a long time ago in Ref. [37] proposing the difference in the transverse energy distribution of leptons and antileptons from $t \bar{t}$ events at hadron colliders as probe of $C P$ violation in the Higgs sector. A previous study [38] has also already touched upon this idea. The authors consider $t \bar{t}$ production at the LHC and allow rescaling the $C P$-even Yukawa coupling in a pre-existing SM calculation. An actual measurement by CMS [39] shows very promising sensitivity that warrants further investigation. In contrast, our work requires calculating the respective Higgs boson loops from scratch due to the new $C P$-odd components. We present a realistic phenomenological analysis for the most general $C P$-even and $C P$-odd coupling structure, for the first time, and estimate the sensitivity using the same observables as in the CMS analysis in Ref. [39]. In addition, we simulate the competing on-shell processes $p p \rightarrow t \bar{t} H, p p \rightarrow t q H$, and $p p \rightarrow t W H$ with the same $C P$ even and $C P$-odd top quark Yukawa couplings in order to have a fair comparison and to capture dominant backgrounds. We partly resort to our previous work in Ref. [40] and extend it by the calculation of the $p p \rightarrow t W H$ process, which we discuss in more detail. As a result, all on-shell processes are publicly available ${ }^{1}$ in the JHUGen Monte-Carlo generator [40-44], which is heavily used in experimental analysis $[4,26,45-48]$. We also provide a publicly available $^{2}$ extension of MCFM [49,50], which yields the loop correction to $t \bar{t}$ production. Finally, we note that our implementation also allows for the most general $C P$-even and $C P$-odd $H W W$ anomalous couplings in the $t W H$ process. For the purpose of this work, however, we keep them at their SM value. Recently, the combination of all onshell processes has also been studied in Ref. [51]. Relevant works on a subset of these processes can be found in Refs. [52-62]. Also low-energy measurements of the electric dipole moment yield complementary constraints on the $C P$-odd components, which are remarkably strong [63-67] and need to be considered in real data analyses.

\section{LOOP SENSITIVITY TO THE CP STRUCTURE OF THE TOP-HIGGS COUPLING}

\section{A. The next-to-leading order (NLO) electroweak effects}

The dependence of the $t \bar{t}$ production cross section on the top quark Yukawa coupling arises only when considering electroweak loop corrections. For the SM hypothesis, theoretical predictions for top quark pair production

\footnotetext{
${ }^{1}$ https://spin.pha.jhu.edu.

${ }^{2}$ https://github.com/TOPAZdevelop/MCFM-8.3_EWSMEFT_ ADDON.
}

including electroweak corrections have been known for a long time [38,68-72] and their implementation is available via published codes like MCFM [49]. Version 2.1 of HATHOR [73] allows for the calculation of electroweak corrections to top quark pair production with a scalable $C P$-even top quark Yukawa coupling.

In Ref. [74] the calculation of electroweak loops in hadronic $t \bar{t}$ production with modified couplings of the top quark to the electroweak gauge bosons in terms of higherdimensional EFT operators has been presented by some of us. Building upon the techniques developed in Ref. [74], we allow for arbitrary $C P$ scenarios of the top quark Yukawa couplings parametrized by the effective Lagrangian of the interaction of the top quark $t$ and a scalar particle $H$

$$
\mathcal{L}(H t t)=-\frac{m_{t}}{v} \bar{\psi}_{t}\left(\kappa+\mathrm{i} \tilde{\kappa} \gamma_{5}\right) \psi_{t} H
$$

where the $\kappa$ term is $C P$ even, and the $\tilde{\kappa}$ term is $C P$ odd. The parameters $\kappa$ and $\tilde{\kappa}$ can be connected to the real and imaginary part of the Wilson coefficient $C_{t t}^{u \varphi}$ of the respective dimension-six operator $Q_{u \varphi}$, as defined in the Warsaw basis of the Standard Model Effective Field Theory (SMEFT) [75], by

$$
\begin{aligned}
& \kappa=1-\frac{v}{\sqrt{2} m_{t}} \frac{v^{2}}{\Lambda^{2}} \operatorname{Re}\left[C_{t t}^{u \varphi}\right], \\
& \tilde{\kappa}=-\frac{v}{\sqrt{2} m_{t}} \frac{v^{2}}{\Lambda^{2}} \operatorname{Im}\left[C_{t t}^{u \varphi}\right] .
\end{aligned}
$$

This effective Lagrangian incorporates additional $C P$-odd states, inherent to, e.g., SUSY or two-Higgs-doublet models, while allowing for arbitrary $C P$ mixing with $C P$-even states, eventually recovering the SM for $\kappa=1$ and $\tilde{\kappa}=0$ (cf. Ref. [76]). We employ the Feynman rules implied by the Lagrangian in Eq. (1) to calculate predictions for top quark pair production including electroweak corrections while parametrizing arbitrary $C P$ scenarios of the top quark Yukawa coupling by $\kappa$ and $\tilde{\kappa}$.

Figure 1 shows sample diagrams for the production of a top quark pair in $q \bar{q}$ annihilation or gluon fusion including a Higgs boson running in the loop. The final state corrections shown in Fig. 1(a) apply to both gluonic and quark-antiquark $s$-channel production. Due to the scalar and pseudoscalar contributions to the top quark Yukawa coupling in Eq. (1), the interference terms of the tree level with the Higgs-loop diagrams are solely comprised of terms proportional to $\left(\kappa^{2}+\tilde{\kappa}^{2}\right)$ or $\left(\kappa^{2}-\tilde{\kappa}^{2}\right)$ when neglecting the masses of the light quarks. These terms are infrared finite but contain UV divergences. Thus, the renormalization of the top quark wave function and the top quark mass has to be consistently performed with the modified top quark Yukawa coupling in order to ensure the cancellation of the 


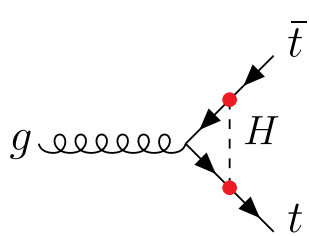

(a)

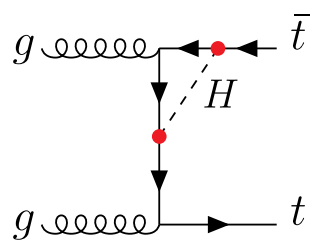

(c)

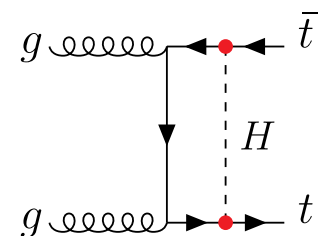

(b)

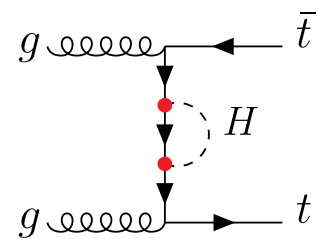

(d)
FIG. 1. Exemplary weak corrections from Higgs boson exchange to $t \bar{t}$ production: Final-state vertex correction affecting the $s$-channel both in $q \bar{q}$ annihilation and gluon fusion (a). Box diagram (b), vertex correction (c), and self-energy corrections (d) to the $t$-channel in gluon fusion.

UV divergences for arbitrary values of $\kappa$ and $\tilde{\kappa}$. Following Ref. [77], we write the bare top quark field $t_{0}=(1+$ $\left.\frac{1}{2} \delta Z_{t}\right) t$ and the bare top quark mass $m_{0}=m+\delta m_{t}$ in terms of the respective renormalized quantities $t$ and $m$ together with the renormalization constants

$$
\begin{aligned}
\delta Z_{t}= & -\operatorname{Re}\left[\Sigma_{t}^{\mathrm{L}}\left(m_{t}^{2}\right)+\Sigma_{t}^{\mathrm{R}}\left(m_{t}^{2}\right)\right] \\
& -\left.2 m_{t}^{2} \frac{\partial}{\partial p^{2}} \operatorname{Re}\left[\Sigma_{t}^{\mathrm{L}}\left(p^{2}\right)+\Sigma_{t}^{\mathrm{R}}\left(p^{2}\right)+2 \Sigma_{t}^{\mathrm{S}}\left(p^{2}\right)\right]\right|_{p^{2}=m_{t}^{2}}, \\
\delta m_{t}= & \frac{m_{t}}{2} \operatorname{Re}\left[\Sigma_{t}^{\mathrm{L}}\left(m_{t}^{2}\right)+\Sigma_{t}^{\mathrm{R}}\left(m_{t}^{2}\right)+2 \Sigma_{t}^{\mathrm{S}}\left(m_{t}^{2}\right)\right] .
\end{aligned}
$$

The terms $\Sigma_{t}^{\lambda}\left(p^{2}\right),(\lambda=\mathrm{L}, \mathrm{R}, \mathrm{S})$ are the chiral self energies calculated at one-loop order with the electroweak gauge bosons and the Higgs boson running in the loop. In the Higgs sector, they receive modifications with respect to the SM when arbitrary $C P$ scenarios of the top quark Yukawa coupling are taken into account

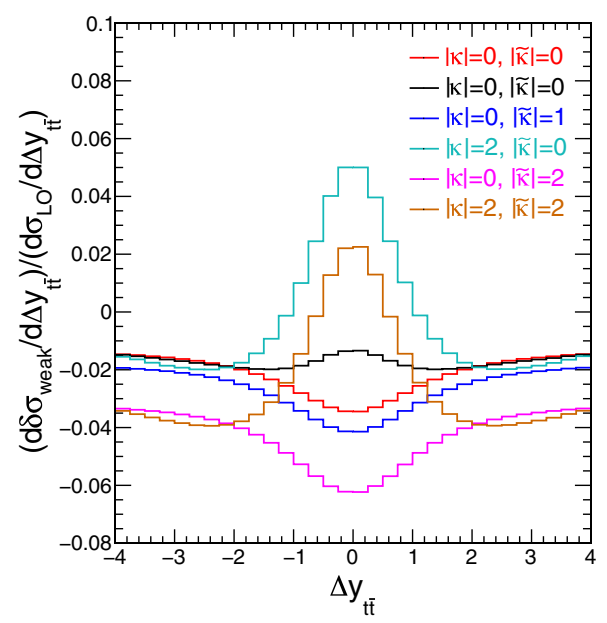

$$
\begin{aligned}
& \Sigma_{t H}^{\mathrm{L}}=\Sigma_{t H, \mathrm{SM}}^{\mathrm{L}}\left(\kappa^{2}+\tilde{\kappa}^{2}\right), \\
& \sum_{t H}^{\mathrm{R}}=\Sigma_{t H, \mathrm{SM}}^{\mathrm{R}}\left(\kappa^{2}+\tilde{\kappa}^{2}\right), \\
& \Sigma_{t H}^{\mathrm{S}}=\Sigma_{t H, \mathrm{SM}}^{\mathrm{S}}\left(\kappa^{2}-\tilde{\kappa}^{2}\right) .
\end{aligned}
$$

After renormalization, the one-loop amplitude is UV finite for arbitrary values of $\kappa$ and $\tilde{\kappa}$ and remains without any dependence on interference terms proportional to $\kappa \tilde{\kappa}$. However, because of different contributions proportional to $\left(\kappa^{2}+\tilde{\kappa}^{2}\right)$ as well as $\left(\kappa^{2}-\tilde{\kappa}^{2}\right)$, the shapes of kinematic distributions are separately sensitive to $\kappa$ and $\tilde{\kappa}$.

We build upon the existing implementation of the electroweak corrections to top quark pair production in MCFM [49] and modify the code by the analytic results of the calculation outlined above. This extension is publicly available as an external add-on to the MCFM program. With the modified Monte-Carlo generator MCFM, the relative corrections to the LO result

$$
\delta_{\mathrm{wk}}=\frac{d \sigma_{\mathrm{wk}}^{\mathrm{NLO}}-d \sigma^{\mathrm{LO}}}{d \sigma^{\mathrm{LO}}}
$$

can be calculated for multidimensional kinematic distributions dependent on $\kappa$ and $\tilde{\kappa}$. Following Ref. [39], where the size of a pure $C P$-even top Yukawa coupling was measured through the distributions of the invariant mass of the top quark pair $M_{t \bar{t}}$ and their rapidity difference, $\Delta y_{t \bar{t}}=y_{t}-y_{\bar{t}}$, we show the electroweak correction factor for these distributions in Fig. 2. The rapidity difference shows a strong dependence on the $C P$ structure of the top quark Yukawa coupling: The $C P$-even contribution increases the central region while the $C P$-odd contribution decreases it. The invariant mass of the top quark pair shows dependence on the $C P$ structure in the threshold region as well as in the tail. Therefore, these kinematic distributions are promising candidates for probing the size of possible $C P$ mixtures.

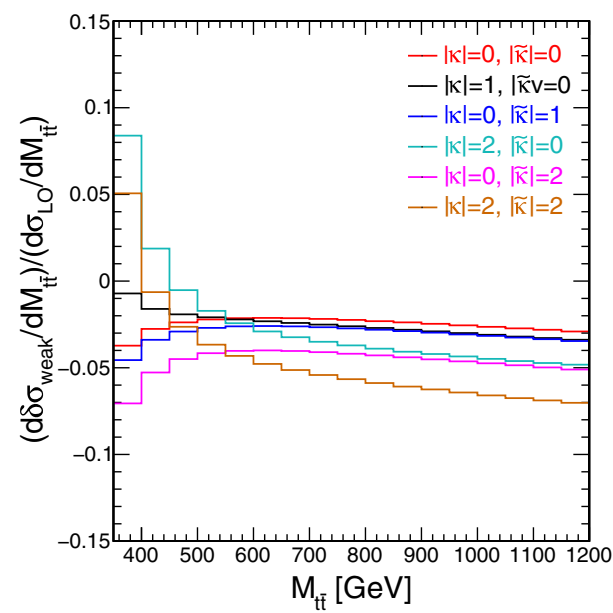

FIG. 2. The ratio of weak corrections over the LO $t \bar{t}$ production cross section varies with the sensitive kinematic variables $\Delta y_{t \bar{t}}$ (left) and $M_{t \bar{t}}$ (right) on generator level for different anomalous parameters $\kappa$ and $\tilde{\kappa}$. The black lines correspond to the SM case. 


\section{B. Expected sensitivity through top quark pair production}

To avoid complicated combinatorial issues, we perform the study in the semileptonic channel, where one top quark decays hadronically and the other decays leptonically. This final state consists of one lepton (electron or muon), missing transverse momentum, four jets from two bottom quarks and two light-flavor quarks. The main background comes from single top, $V+$ jets and QCD multijets processes. For simplicity, we simulate the single top process to extract the shape and rescale it to the expectation of all background processes according to the results presented in the CMS analysis [39].

In this study, the events are simulated by MadGraph5_v2.6.4 [78] and interfaced to Pythia8.1 [79] for parton shower. The detector simulation is implemented by Delphes3 [80] with the CMS detector setting. The NLO weak effects are incorporated in the generated LO events by performing a twodimensional reweighting. The weights are obtained from the above calculation, as implemented in our publicly available extension of the MCFM program, in the $M_{t \bar{t}}-$ $\Delta y_{t \bar{t}}$ phase space, and applied to the LO events based on their truth level information. The simulated events are normalized to the $t \bar{t}$ production cross section of $\sigma_{t \bar{t}}=$ $832_{-46}^{+40} \mathrm{pb}[32,81,82]$ predicted at next-to-next-to-leading order (NNLO) QCD accuracy. The cross section of single top quark production is normalized to the NLO QCD prediction $[83,84]$.

Jets and leptons with $p_{T}>30 \mathrm{GeV}$ and $|\eta|<2.4$ are selected. Events are required to have four jets and exactly one lepton. Two of the four jets should be $b$-tagged jets. For the $W$ boson that decays leptonically, its transverse mass $M_{\mathrm{T}}(W)$ is required to be less than $140 \mathrm{GeV} . M_{\mathrm{T}}(W)$ is defined as $M_{\mathrm{T}}(W)=\sqrt{2 p_{\mathrm{T}}^{\ell} p_{\mathrm{T}}^{\text {miss }}\left[1-\cos \left(\Delta \phi_{\ell, \vec{p}_{\mathrm{T}}^{\text {miss }}}\right)\right]}$, where $p_{\mathrm{T}}^{\ell}$ is the magnitude of the transverse momentum of the

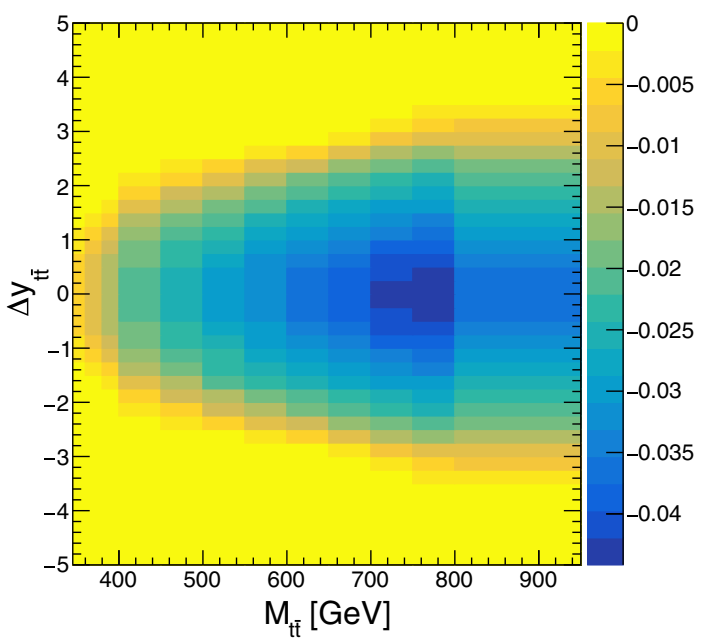

lepton and $\vec{p}_{\mathrm{T}}^{\text {miss }}$ denotes the missing transverse momentum. The other $W$ boson is reconstructed from the two light flavor jets. Top quark pairs are reconstructed through a maximum likelihood method [85].

The ratio of the weak corrections over the LO cross section in the two-dimensional plane of $M_{t \bar{t}}$ and $\Delta y_{t \bar{t}}$ at parton level is shown in Fig. 3. The SM couplings $(\kappa=1$, $\tilde{\kappa}=0$ ) are used. The corrections illustrate a correlation pattern between the two variables, thus it is important to perform the analysis in 2D. The number of events in the 2D space after event selection and reconstruction are shown in the right plot of Fig. 3 .

Nonzero $\kappa$ and $\tilde{\kappa}$ values would distort the event distributions as shown in Fig. 3, and the effects would propagate to the reconstructed kinematics. The amount of $C P$ violation of the top quark Yukawa coupling can be quantified by the parameter

$$
f_{\mathrm{CP}}=\frac{|\tilde{\kappa}|^{2}}{|\kappa|^{2}+|\tilde{\kappa}|^{2}} \operatorname{sign}\left(\frac{\tilde{\kappa}}{\kappa}\right),
$$

which is naturally restricted to values from -1 to 1 . Its absolute value represents the fractional size of the $C P$-odd component, and its sign reflects the relative phase of the two couplings. We use the reconstructed 2D distribution of $M_{t \bar{t}}$ and $\Delta y_{t \bar{t}}$ to extract the value of the $C P$ mixture parameter $f_{\mathrm{CP}}$. A profile likelihood method is used to obtain the expected sensitivity at $300 \mathrm{fb}^{-1}$ and $3000 \mathrm{fb}^{-1}$, respectively. Theoretical uncertainties of the signal and background processes are taken into account. In particular, QCD uncertainties of the $t \bar{t}$ process at NNLO are about 5\% in the most relevant regions of the $\Delta y_{t \bar{t}}$ and $M_{t \bar{t}}$ distributions [32]. Thus, we assign an overall 5\% uncertainty to the $t \bar{t}$ rate. The size of the uncertainty is found to have little impact on the results. The details of the results will be discussed in Sec. IV.

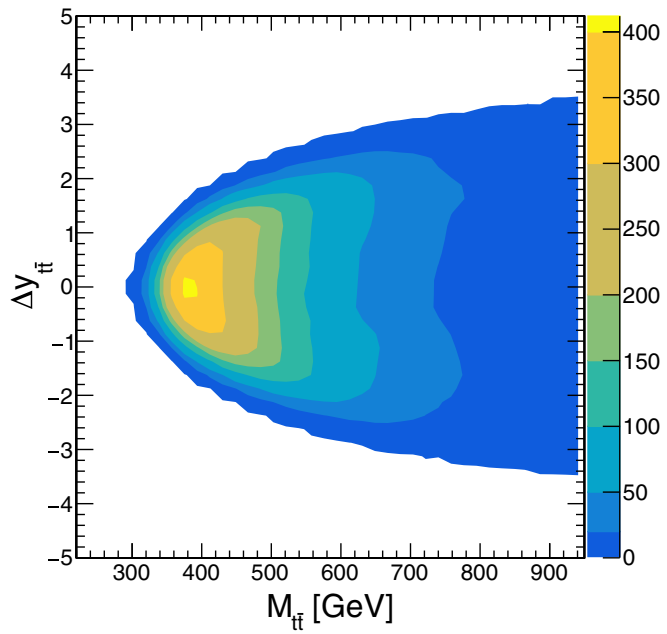

FIG. 3. Left: The size of NLO weak correction over LO prediction in the two-dimensional $M_{t \bar{t}}$ and $\Delta y_{t \bar{t}}$ plane, obtained at truth level with $\kappa=1$ and $\tilde{\kappa}=0$. Right: Number of simulated events in the two-dimensional plane of $M_{t \bar{t}}$ and $\Delta y_{t \bar{t}}$ at reconstruction level. 


\section{ON-SHELL SENSITIVITY TO THE $C P$ STRUCTURE OF THE TOP-HIGGS COUPLING}

\section{A. Higgs production in association with a single top quark}

The associated production of a single top quark or a top quark pair with a Higgs boson is dependent on the top quark Yukawa coupling at tree level already. Respective analyses for hadronic $t \bar{t} H$ and $t q H$ production are presented in Ref. [40]. For this work, we complete the existing results by also considering the associated production of a single top quark with a $W$ and a Higgs boson. The $t W H$ production at tree level has two categories of Feynman diagrams as shown in Fig. 4. One category is induced by the Htt coupling and the other is induced by the $H W W$ coupling. These two categories of Feynman diagrams interfere destructively in the SM, which leads to a small total cross section of about $17 \mathrm{fb}$ in the SM. However, $C P$ violation would increase the total cross section especially when the relative sign of the $H t t$ and $H W W$ couplings flips. Single top quark production in association with a Higgs boson is also sensitive to the relative sign of the Htt and $H W W$ couplings due to these interference terms. To consider arbitrary $C P$ scenarios for the top quark Yukawa coupling we use again the Feynman rules implied by the Lagrangian in Eq. (1) to calculate theoretical predictions for the $t W H$ production. We also include anomalous $H W W$ couplings by following the notation of Refs. [41-43] to parametrize the Lagrangian for the interaction of a scalar $H$ and two $W$ bosons

$$
\begin{aligned}
\mathcal{L}(H W W)= & \frac{M_{W}^{2}}{v}\left[g_{1}^{W W} W_{\mu}^{+} W_{\mu}^{-}-\frac{g_{2}^{W W}}{M_{W}^{2}} W_{\mu \nu}^{+} W_{\mu \nu}^{-}\right. \\
& +\frac{\kappa_{1}^{W W}}{\left(\Lambda_{1}^{W W}\right)^{2}}\left(W_{\mu}^{-} \partial_{\nu} W_{\mu \nu}^{+}+\text {H.c. }\right) \\
& \left.-\frac{g_{4}^{W W}}{M_{W}^{2}} W_{\mu \nu}^{+} \tilde{W}_{\mu \nu}^{-}\right] H .
\end{aligned}
$$

Again, the coupling parameters above have direct relations to Wilson coefficients of corresponding dimensionsix operators in the Warsaw basis of the SMEFT (cf. Refs. [44,86]). Our results are incorporated in the JHUGen thereby completing the framework's

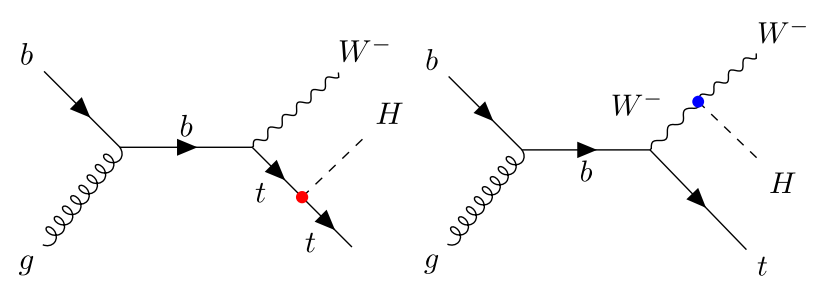

FIG. 4. Two typical Feynman diagrams of the $t H W$ production at tree level. The Htt induced process (left) interferes with the $H W W$ induced process (right). implementation of single top quark production in association with a Higgs boson with anomalous $\mathrm{Htt}$ and $\mathrm{HWW}$ couplings. In this paper, the $t W H$ production includes both the top quark associated process and the antitop quark associated process and the $H W W$ couplings are set to their SM values.

\section{B. Expected sensitivity through Higgs production in association with a single top quark}

We estimate the expected constraints on the top-Higgs $C P$ property in the $t W H$ process using a matrix element method. The matrix element likelihood approach (MELA) is designed to extract all essential information from the complex kinematics of a given final state. It can transform complex kinematics into a minimal set of discriminants calculated from the ratios of the matrix elements. To distinguish two different hypotheses, the ratio of probabilities $\mathcal{P}$ for the two hypotheses offers an optimal tool according to the Neyman-Pearson lemma [87]. For measurements of properties of the Higgs boson, two types of discriminants $[40,43]$ defined as below have proven to be useful

$$
\begin{aligned}
& \mathcal{D}_{\text {alt }}=\frac{\mathcal{P}_{\mathrm{A}}(\vec{\Omega})}{\mathcal{P}_{\mathrm{A}}(\vec{\Omega})+\mathcal{P}_{\mathrm{B}}(\vec{\Omega})}, \\
& \mathcal{D}_{\text {int }}=\frac{\mathcal{P}_{\text {int }}(\vec{\Omega})}{\mathcal{P}_{\mathrm{A}}(\vec{\Omega})+\mathcal{P}_{\mathrm{B}}(\vec{\Omega})},
\end{aligned}
$$

where $\vec{\Omega}$ represents the 4-momenta of all particles in each final state. The probability densities $\mathcal{P}$ under certain hypotheses $\mathrm{A}$ and $\mathrm{B}\left(\mathcal{P}_{\mathrm{A}}(\vec{\Omega})\right.$ and $\left.\mathcal{P}_{\mathrm{B}}(\vec{\Omega})\right)$ for each event are calculated through the squared matrix element. Parton distribution functions have to be taken into account in the calculation when multiple initial parton states are concerned. $\mathcal{D}_{\text {alt }}$ is useful to disentangle hypotheses $\mathrm{A}$ and $\mathrm{B}$, while $\mathcal{D}_{\text {int }}$, using the interference probability density $\mathcal{P}_{\text {int }}(\vec{\Omega})$ between two hypotheses, is sensitive to the interference effect.

TABLE I. Cross sections and expected number of events for signal and other contributions at a luminosity of $300 \mathrm{fb}^{-1}$ at $13 \mathrm{TeV}$. Here, $C P$-even corresponds to $\kappa=1$ and $\tilde{\kappa}=0$, while $C P$-odd corresponds to $\kappa=0$ and $\tilde{\kappa}=1$. The expected numbers of events are reported after event selection in the $H \rightarrow \gamma \gamma$ final state.

\begin{tabular}{lcc}
\hline \hline Process & Cross section [fb] Expected number of events \\
\hline$t W H(C P$-even $)$ & 16.8 & 0.72 \\
$t W H(C P$-odd $)$ & 69.5 & 3.99 \\
$t \bar{t} H(C P$-even $)$ & 509.0 & 16.91 \\
$t \bar{t} H$ ( $C P$-odd $)$ & 198.5 & 8.21 \\
\hline \hline
\end{tabular}



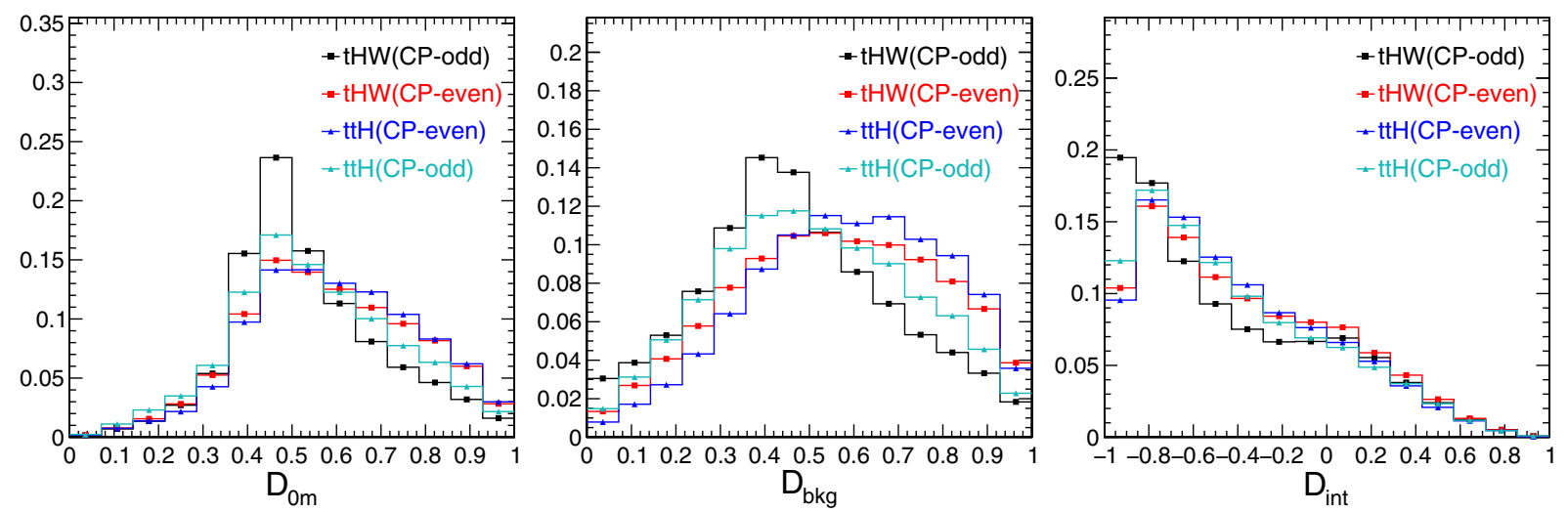

FIG. 5. The distributions of $D_{0 \mathrm{~m}}, D_{\mathrm{bkg}}$, and $D_{\text {int }}$. Four different scenarios are presented: the $p p \rightarrow t W H$ process with $C P$-even or $C P$-odd Yukawa couplings and the $p p \rightarrow t \bar{t} H$ process with $C P$-even or $C P$-odd Yukawa couplings.

To estimate the sensitivity in the $p p \rightarrow t W H$ process, we consider hadronic final states, where both the top quark and $W$ boson decay hadronically, and $H \rightarrow \gamma \gamma$. This final state has a reasonable branching ratio and clean boson decays. The main background process is $p p \rightarrow t \bar{t} H$. Both $t \bar{t} H$ and $t W H$ production are sensitive to the top quark Yukawa coupling, and the predicted cross sections at tree level are functions of $\kappa$ and $\tilde{\kappa}$,

$$
\begin{aligned}
\sigma(\kappa, \tilde{\kappa})_{t \bar{t} H} & =\sigma_{\mathrm{SM}}^{t \bar{t} H}\left(|\kappa|^{2}+0.39|\tilde{\kappa}|^{2}\right), \\
\sigma(\kappa, \tilde{\kappa})_{t W H} & =\sigma_{\mathrm{SM}}^{t W H}\left(2.82|\kappa|^{2}+2.08|\tilde{\kappa}|^{2}-3.87 \kappa+2.05\right),
\end{aligned}
$$

where $\sigma_{\mathrm{SM}}^{t \bar{t} H}$ and $\sigma_{\mathrm{SM}}^{t W H}$ are the SM cross sections of $t \bar{t} H$ and $t W H$ production, respectively.

We simulate $t W H$ and $t \bar{t} H$ events using JHUGen. The parton shower and hadronization are implemented by Pythia8.1 and Delphes3 is used to simulate the CMS detector response.

For event selection, we require at least 5 jets with $p_{T}>$ $25 \mathrm{GeV}$ and $|\eta|<2.4$, and exactly one $b$-tagged jet. Events with any isolated leptons or more than 8 jets are vetoed to remove $\bar{t} \bar{t} H$ contributions. Two isolated photons are needed to pass the event selection criteria. The transverse momenta of the leading and subleading photons are required to have $p_{\mathrm{T}}^{1}>m_{\gamma \gamma} / 3$ and $p_{\mathrm{T}}^{2}>m_{\gamma \gamma} / 4$, respectively. To further suppress the $t t H$ background, we require the transverse momentum of the Higgs boson to satisfy $p_{\mathrm{T}}^{H}>80 \mathrm{GeV}$. The expected number of events of the signal and background processes at $300 \mathrm{fb}^{-1}$ after selection are summarized in Table I. Other non-Higgs background processes like $t t+\gamma \gamma$ may be distinguished by the invariant mass of the reconstructed Higgs boson, thus are not taken into account in this study.

We apply similar techniques as used in Sec. II to reconstruct the top quark in the final state. In total we build four matrix-element based discriminants: $\mathcal{D}_{0 \mathrm{~m}}, \mathcal{D}_{\text {bkg }}$, $\mathcal{D}_{\mathrm{CP}}$, and $\mathcal{D}_{\text {int }}$. The first two variables are of $\mathcal{D}_{\text {alt }}$ type as defined in Eq. (5). Model $\mathrm{A}$ and $\mathrm{B}$ are $C P$-even and
$C P$-odd model in $\mathcal{D}_{0 \mathrm{~m}}$, and are pure $\mathrm{Htt}$ diagram contribution and pure $H W W$ contribution in $\mathcal{D}_{\text {bkg }}$, respectively. $\mathcal{D}_{\mathrm{CP}}$ and $D_{\text {int }}$ are interference sensitive variables following the defintion given in Eq. (6). $\mathcal{D}_{\mathrm{CP}}$ is designed to detect the interference between $\mathrm{CP}$-even and $\mathrm{CP}$-odd $\mathrm{Htt}$ couplings, and $\mathcal{D}_{\text {int }}$ is to obtain the interference between $H W W$ and Htt couplings. The distributions of $\mathcal{D}_{0 \mathrm{~m}}, \mathcal{D}_{\text {bkg }}$, and $\mathcal{D}_{\text {int }}$ after event selection and reconstruction are presented in Fig. 5. The $C P$-even $(\kappa=1)$ and $C P$-odd $(\tilde{\kappa}=1)$ scenarios of $t W H$ and $t \bar{t} H$ production are shown. $C P$-even and $C P$-odd $t W H$ and $t \bar{t} H$ production are well separated in $\mathcal{D}_{0 \mathrm{~m}}$ and $D_{\mathrm{bkg}}$, and $D_{\text {int }}$ is sensitive to the interference term of the $t W H$ production. We use $\mathcal{D}_{0 \mathrm{~m}}, \mathcal{D}_{\text {bkg }}$, and $\mathcal{D}_{\text {int }}$ to construct a 3-dimensional probability density function, which is fitted to the $\mathrm{SM}$ distribution to estimate the $C P$ sensitivity. $D_{\mathrm{CP}}$ is only forward-backward asymmetric in models with $C P$ violation as shown in shown in Fig. 6, thus not used for the SM sensitivity estimation. However it will be a very

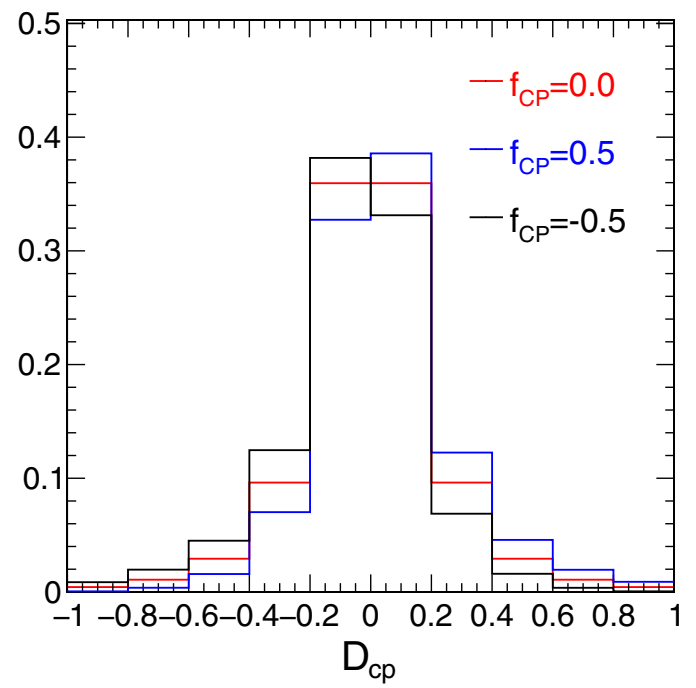

FIG. 6. The distribution of $\mathcal{D}_{\mathrm{CP}}$ of the $p p \rightarrow t W H$ process in three scenarios: $f_{\mathrm{CP}}=0$ (red), $f_{\mathrm{CP}}=0.5$ (blue), and $f_{\mathrm{CP}}=-0.5$ (black). 
powerful observable to detect any $C P$ violation. Unlike in the $p p \rightarrow t \bar{t} H$ process, without using the decay information of the $W$ boson and the top quark, the forward-backward asymmetry in $\mathcal{D}_{\mathrm{CP}}$ remains in the $t W H$ channel. This advantage of the $p p \rightarrow t W H$ process allows to probe the sign of the $C P$ violation in all top quark and $W$ decay modes.

\section{RESULTS}

A maximum likelihood fit is performed in the $t \bar{t}$ and $t W H$ events to quantify the sensitivity to the $C P$ structure of the top quark Yukawa coupling. In this study, the Higgs boson coupling to other particles except the top quark are constrained to their SM value. The $H \rightarrow \gamma \gamma$ interaction could be modified by the top quark Yukawa coupling if we assume the loop is resolved. However, only the rates of $t W H$ and $t \bar{t} H$ will be affected. As this study focuses on the kinematic effects on the Higgs production, we assume the $H \rightarrow \gamma \gamma$ rate is the same as the SM prediction. We present the results in two forms: one in terms of the Lagrangian coupling parameters $\kappa$ and $\tilde{\kappa}$, and the other in terms of the $C P$-mixture parameter $f_{\mathrm{CP}}$.

The expected likelihood scan results of $\kappa$ and $\tilde{\kappa}$ at $300 \mathrm{fb}^{-1}$ are shown in Fig. 7. The left and middle plots show the sensitivity using $t \bar{t}$ and $t W H$ events, respectively. The right plot shows the expected sensitivity using $t H q$ events, derived from Ref. [40]. It is clear from the middle and right plots that single top quark production provides sensitivity to the relative sign between the $\mathrm{Htt}$ and $H W W$ coupling, while the $t \bar{t}$ plot is symmetric around $\kappa=0$. This is expected as seen from Eqs. (2)-(4).

The sensitivities of the parameter $f_{\mathrm{CP}}$ at the luminosity of $300 \mathrm{fb}^{-1}$ and $3000 \mathrm{fb}^{-1}$ are shown in Fig. 8. When fitting the distributions in the $f_{\mathrm{CP}}$ framework, the overall signal rate is left unconstrained. This means whatever modification might enter $H \rightarrow \gamma \gamma$ is absorbed by this floating rate, thus the $f_{\mathrm{CP}}$ result is not affected by the assumptions in the
$H \rightarrow \gamma \gamma$ decay. The sensitivity using $t \bar{t} H$ and $t q H$ presented in Ref. [40] are also shown for comparison. One should note that while $t H$ dedicated studies aim to select $t H$ events, $t \bar{t} H$ events enter the selection due to similar final state particles. These events contribute to the $C P$ sensitivity in the $t H$ channel as well. Without such background, the pure contribution from the $t W H$ events are shown as a dashed line. The curve of $t \bar{t}$ reaches a plateau around $\left|f_{\mathrm{CP}}\right|=0.87$. This is where a switch between shape and rate effect comes into place. Beyond the boundary, the dominant effect is the overall change in the event rate with little kinematic shape variations, thus absorbed by the floating rate parameter.

The expected sensitivities show that $t \bar{t}$ and $t H$ events are prone to different phase spaces. They could be complementary to each other in constraining the $C P$ violation in top quark Yukawa coupling. Up to $300 \mathrm{fb}^{-1}$, they provide rather compatible $95 \%$ CL constraints. It will be interesting to see experimental results using all the processes.

The $p p \rightarrow t \bar{t}$ process is expected to exclude $\left|f_{\mathrm{CP}}\right|>0.81$ at $95 \% \mathrm{CL}$ at the luminosity of $300 \mathrm{fb}^{-1}$. Although the total cross section of the $t W H$ production in the SM is small, compared with the $t \bar{t} H$ and $t q H$ production, this process can still exclude $\left|f_{\mathrm{CP}}\right|>0.68$ at $68 \% \mathrm{CL}$ and exclude the pure pseudoscalar model at $2 \sigma$ at a luminosity of $300 \mathrm{fb}^{-1}$. The results at a luminosity of $300 \mathrm{fb}^{-1}$ can easily be projected to other luminosities such as $3000 \mathrm{fb}^{-1}$ at the HL-LHC. At a luminosity of $3000 \mathrm{fb}^{-1}$, the $p p \rightarrow t W H$ process can exclude $\left|f_{\mathrm{CP}}\right|>0.48$ at $95 \% \mathrm{CL}$, and $\left|f_{\mathrm{CP}}\right|>$ 0.67 can be excluded by the $p p \rightarrow t \bar{t}$ process at 95\% CL. Among the four processes, the $t q H$ production, together with the $t \bar{t} H$ events entering the selection, gives most stringent $95 \%$ CL exclusion, which can exclude $\left|f_{\mathrm{CP}}\right|>$ 0.68 at a luminosity of $300 \mathrm{fb}^{-1}$ and $\left|f_{\mathrm{CP}}\right|>0.22$ at a luminosity of $3000 \mathrm{fb}^{-1}$. For values of $\left|f_{\mathrm{CP}}\right|>0.8$, the $p p \rightarrow t \bar{t}$ process is the best candidate for exclusion at a luminosity of $3000 \mathrm{fb}^{-1}$.
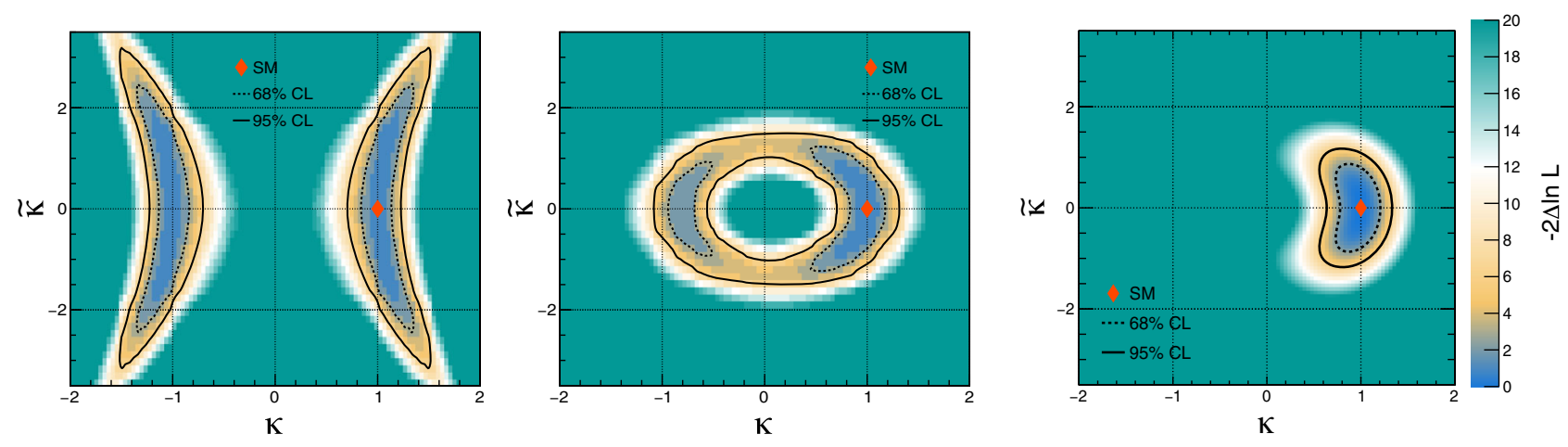

FIG. 7. Two dimensional likelihood scans of $\kappa$ and $\tilde{\kappa}$ in the $p p \rightarrow t \bar{t}$ (left) and $p p \rightarrow t W H$ (middle) and $p p \rightarrow t q H$ (right) processes at a luminosity of $300 \mathrm{fb}^{-1}$. The expected $68 \%$ and $95 \%$ CL regions are presented as contours with dashed and solid black lines, respectively. 

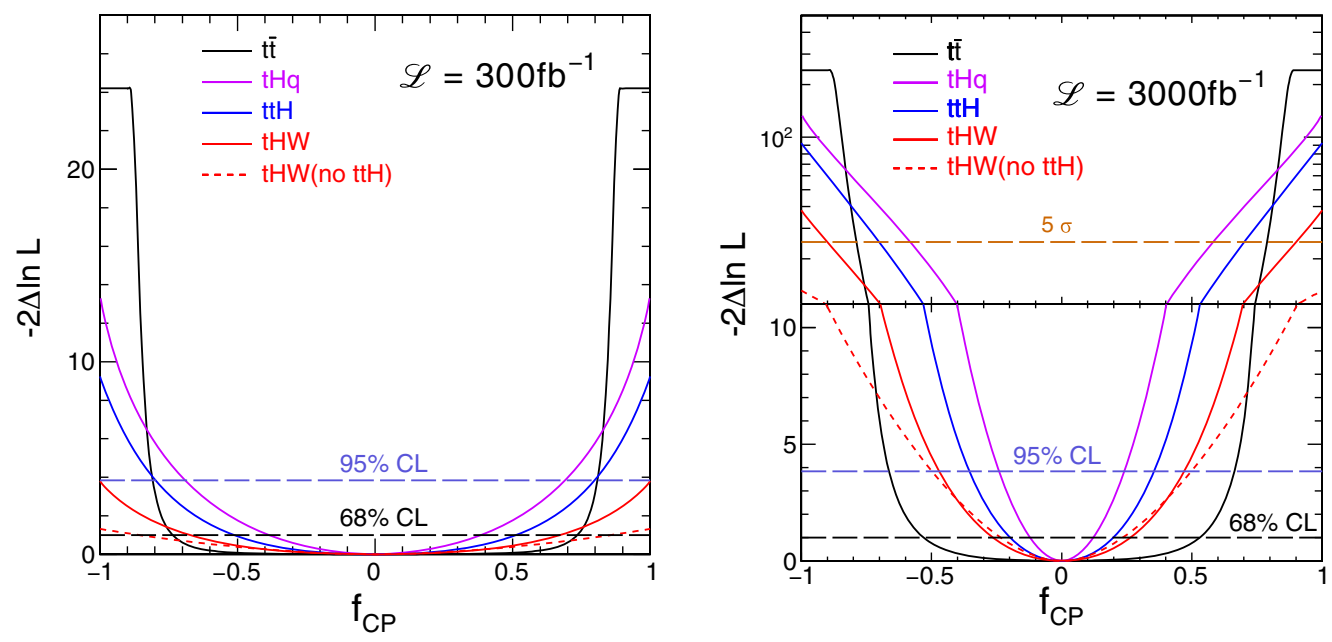

FIG. 8. The likelihood scan of $f_{\mathrm{CP}}$ at luminosity of $300 \mathrm{fb}^{-1}$ (left) and $3000 \mathrm{fb}^{-1}$ (right) with four processes shown: $p p \rightarrow t \bar{t}$, $p p \rightarrow t W H, p p \rightarrow t \bar{t} H$, and $p p \rightarrow t q H$. The red solid line shows the expectation considering $t W H$ and the misreconstructed $t t H$ events in the $t W H$ channel, while the red dashed line assumes no contribution of $t t H$ events in the $t W H$ channel. The black and dark blue lines represent the $68 \% \mathrm{CL}$ and $95 \% \mathrm{CL}$ lines, respectively. The results of the $t \bar{t} H$ and $t q H$ production are cited from Ref. [40].

\section{SUMMARY}

In this paper, we investigate the prospects of constraining the $C P$ structure of the coupling of the Higgs boson to the top quark through electroweak loops in $t \bar{t}$ production. Sensitivity arises at loop level through off-shell degrees of freedom only. The fact that top quark pair production is the most dominant source of top quarks at the LHC while its theoretical description has reached impressive accuracy makes it the perfect candidate for such a novel study. To this end, we calculate $\mathcal{O}(\alpha)$ corrections to QCD induced $t \bar{t}$ production with arbitrary $C P$-even and $C P$-odd terms. Our results for the loop sensitivity are contrasted with the ones obtained from direct on-shell probes like Higgs production in association with a single top quark or a top quark pair. Our results show that loop sensitivity in $t \bar{t}$ is significantly stronger than on-shell sensitivity in associated production for $C P$-odd admixtures $|\tilde{\kappa}| /|\kappa| \geq 2$. 0 . Below that value, the $p p \rightarrow t q H, p p \rightarrow t \bar{t} H$, and $p p \rightarrow t H W$ processes are more sensitive. We hope that this work demonstrates the power of loop corrections and sparks new studies. For example, in order to improve on modeling the impact of the electroweak corrections on the full kinematics in $t \bar{t}$ production, our results could be implemented in an event generator like JHUGen. This would allow for generation of unweighted events including electroweak corrections together with the respective weights for different Beyond Standard Model (BSM) hypotheses, making loop sensitivity studies possible with matrix element techniques like MELA. In addition, it would be interesting to extend our calculation to $e^{+} e^{-} \rightarrow t \bar{t}$ for future collider studies.

\section{ACKNOWLEDGMENTS}

We thank Andrei Gritsan for carefully reading the manuscript and his valuable feedback. We thank Mingtao Zhang for the help on validating the event generators and producing useful inputs to the study. We thank Klaus Mönig for valuable feedback on the implementation. This work was supported by the Fundamental Research Funds for the Central Universities (China).
[1] CMS Collaboration, Observation of a new boson at a mass of $125 \mathrm{GeV}$ with the CMS experiment at the LHC, Phys. Lett. B 716, 30 (2012).

[2] ATLAS Collaboration, Observation of a new particle in the search for the standard model Higgs boson with the ATLAS detector at the LHC, Phys. Lett. B 716, 1 (2012).
[3] CMS Collaboration, Observation of a new boson with mass near $125 \mathrm{GeV}$ in $p p$ collisions at $\sqrt{s}=7$ and $8 \mathrm{TeV}$, J. High Energy Phys. 06 (2013) 081.

[4] CMS Collaboration, Constraints on the spin-parity and anomalous HVV couplings of the Higgs boson in proton collisions at 7 and 8 TeV, Phys. Rev. D 92, 012004 (2015). 
[5] ATLAS and CMS Collaborations, Combined Measurement of the Higgs Boson Mass in $p p$ Collisions at $\sqrt{s}=7$ and $8 \mathrm{TeV}$ with the ATLAS and CMS Experiments, Phys. Rev. Lett. 114, 191803 (2015).

[6] I. F. Ginzburg and M. Krawczyk, Symmetries of two Higgs doublet model and CP violation, Phys. Rev. D 72, 115013 (2005).

[7] J. F. Gunion and H. E. Haber, Conditions for $C P$-violation in the general two-Higgs-doublet model, Phys. Rev. D 72, 095002 (2005).

[8] M. Maniatis, A. von Manteuffel, and O. Nachtmann, $C P$ violation in the general two-Higgs-doublet model: A geometric view, Eur. Phys. J. C 57, 719 (2008).

[9] G. C. Branco, P. M. Ferreira, L. Lavoura, M. N. Rebelo, M. Sher, and J. P. Silva, Theory and phenomenology of twoHiggs-doublet models, Phys. Rep. 516, 1 (2012).

[10] H. E. Haber and Z. Surujon, A group-theoretic condition for spontaneous CP violation, Phys. Rev. D 86, 075007 (2012).

[11] D. B. Kaplan, H. Georgi, and S. Dimopoulos, Composite Higgs scalars, Phys. Lett. 136B, 187 (1984).

[12] H. Georgi, D. B. Kaplan, and P. Galison, Calculation of the composite Higgs mass, Phys. Lett. 143B, 152 (1984).

[13] H. Georgi and D. B. Kaplan, Composite Higgs and custodial SU(2), Phys. Lett. 145B, 216 (1984).

[14] M. J. Dugan, H. Georgi, and D. B. Kaplan, Anatomy of a composite Higgs model, Nucl. Phys. B254, 299 (1985).

[15] R. Contino, Y. Nomura, and A. Pomarol, Higgs as a holographic pseudo-Goldstone boson, Nucl. Phys. B671, 148 (2003).

[16] K. Agashe, R. Contino, and A. Pomarol, The minimal composite Higgs model, Nucl. Phys. B719, 165 (2005).

[17] G. F. Giudice, C. Grojean, A. Pomarol, and R. Rattazzi, The strongly-interacting light Higgs, J. High Energy Phys. 06 (2007) 045.

[18] R. Contino, Physics of the large and the small, in TASI 09, proceedings of the Theoretical Advanced Study Institute in Elementary Particle Physics, Boulder, Colorado, USA, 126 June 2009, 2011, pp. 235-306.

[19] S. De Curtis, M. Redi, and A. Tesi, The 4D composite Higgs, J. High Energy Phys. 04 (2012) 042.

[20] M. Redi and A. Weiler, Flavor and $C P$ invariant composite Higgs models, J. High Energy Phys. 11 (2011) 108.

[21] J. Mrazek, A. Pomarol, R. Rattazzi, M. Redi, J. Serra, and A. Wulzer, The other natural two Higgs doublet model, Nucl. Phys. B853, 1 (2011).

[22] M. Redi and A. Tesi, Implications of a light Higgs in composite models, J. High Energy Phys. 10 (2012) 166.

[23] M. Montull, F. Riva, E. Salvioni, and R. Torre, Higgs couplings in composite models, Phys. Rev. D 88, 095006 (2013).

[24] G. Panico and A. Wulzer, The Composite Nambu-Goldstone Higgs (Springer, New York, 2016), Vol. 913.

[25] J. Erdmenger, N. Evans, W. Porod, and K. S. Rigatos, Gauge/Gravity Dynamics for Composite Higgs Models and the Top Mass, Phys. Rev. Lett. 126, 071602 (2021).

[26] CMS collaboration, Measurements of $t \bar{t} H$ Production and the $C P$ Structure of the Yukawa Interaction between the Higgs Boson and Top Quark in the Diphoton Decay Channel, Phys. Rev. Lett. 125, 061801 (2020).
[27] ATLAS Collaboration, $C P$ Properties of Higgs Boson Interactions with Top Quarks in the $t \bar{t} H$ and $t H$ Processes Using $H \rightarrow \gamma \gamma$ with the ATLAS Detector, Phys. Rev. Lett. 125, 061802 (2020).

[28] M. Czakon, P. Fiedler, and A. Mitov, Total Top-Quark PairProduction Cross Section at Hadron Colliders Through $O\left(\alpha_{S}^{4}\right)$, Phys. Rev. Lett. 110, 252004 (2013).

[29] M. Czakon, D. Heymes, and A. Mitov, High-precision Differential Predictions for Top-quark Pairs at the LHC, Phys. Rev. Lett. 116, 082003 (2016).

[30] M. Czakon, P. Fiedler, D. Heymes, and A. Mitov, NNLO QCD predictions for fully-differential top-quark pair production at the tevatron, J. High Energy Phys. 05 (2016) 034.

[31] J. Gao and A. S. Papanastasiou, Top-quark pair-production and decay at high precision, Phys. Rev. D 96, 051501 (2017).

[32] M. Czakon, D. Heymes, A. Mitov, D. Pagani, I. Tsinikos, and M. Zaro, Top-pair production at the LHC through NNLO QCD and NLO EW, J. High Energy Phys. 10 (2017) 186.

[33] A. Behring, M. Czakon, A. Mitov, A. S. Papanastasiou, and R. Poncelet, Higher Order Corrections to Spin Correlations in Top Quark Pair Production at the LHC, Phys. Rev. Lett. 123, 082001 (2019).

[34] C. Muselli, M. Bonvini, S. Forte, S. Marzani, and G. Ridolfi, Top quark pair production beyond NNLO, J. High Energy Phys. 08 (2015) 076.

[35] J. Piclum and C. Schwinn, Soft-gluon and coulomb corrections to hadronic top-quark pair production beyond NNLO, J. High Energy Phys. 03 (2018) 164.

[36] C. Duhr and B. Mistlberger, The N3LO frontier: Precision predictions with QCD perturbation theory, 2021, https://www.snowmass21.org/docs/files/summaries/ TF/SNOWMASS21-TF6_TF4-EF5_EF1-035.pdf.

[37] C. R. Schmidt and M. E. Peskin, A Probe of $C P$ Violation in Top Quark Pair Production at Hadron Supercolliders, Phys. Rev. Lett. 69, 410 (1992).

[38] J. H. Kühn, A. Scharf, and P. Uwer, Weak interactions in top-quark pair production at hadron colliders: An update, Phys. Rev. D 91, 014020 (2015).

[39] CMS Collaboration, Measurement of the top quark Yukawa coupling from $t \bar{t}$ kinematic distributions in the lepton + jets final state in proton-proton collisions at $\sqrt{s}=13 \mathrm{TeV}$, Phys. Rev. D 100, 072007 (2019).

[40] A. V. Gritsan, R. Röntsch, M. Schulze, and M. Xiao, Constraining anomalous Higgs boson couplings to the heavy flavor fermions using matrix element techniques, Phys. Rev. D 94, 055023 (2016).

[41] Y. Gao, A. V. Gritsan, Z. Guo, K. Melnikov, M. Schulze, and N. V. Tran, Spin determination of single-produced resonances at hadron colliders, Phys. Rev. D 81, 075022 (2010).

[42] S. Bolognesi, Y. Gao, A. V. Gritsan, K. Melnikov, M. Schulze, N. V. Tran, and A. Whitbeck, On the spin and parity of a single-produced resonance at the LHC, Phys. Rev. D 86, 095031 (2012).

[43] I. Anderson et al., Constraining anomalous HVV interactions at proton and lepton colliders, Phys. Rev. D 89, 035007 (2014). 
[44] A. V. Gritsan, J. Roskes, U. Sarica, M. Schulze, M. Xiao, and Y. Zhou, New features in the JHU generator framework: Constraining Higgs boson properties from on-shell and offshell production, Phys. Rev. D 102, 056022 (2020).

[45] CMS Collaboration, Combined search for anomalous pseudoscalar HVV couplings in $\mathrm{VH}(\mathrm{H} \rightarrow b \bar{b})$ production and $\mathrm{H} \rightarrow$ VV decay, Phys. Lett. B 759, 672 (2016).

[46] CMS Collaboration, Constraints on anomalous Higgs boson couplings using production and decay information in the four-lepton final state, Phys. Lett. B 775, 1 (2017).

[47] CMS Collaboration, Measurements of the Higgs boson width and anomalous $H V V$ couplings from on-shell and off-shell production in the four-lepton final state, Phys. Rev. D 99, 112003 (2019).

[48] CMS Collaboration, Constraints on anomalous $H V V$ couplings from the production of Higgs bosons decaying to $\tau$ lepton pairs, Phys. Rev. D 100, 112002 (2019).

[49] J. M. Campbell, D. Wackeroth, and J. Zhou, Study of weak corrections to Drell-Yan, top-quark pair, and dijet production at high energies with MCFM, Phys. Rev. D 94, 093009 (2016).

[50] J. Campbell and T. Neumann, Precision phenomenology with MCFM, J. High Energy Phys. 12 (2019) 034.

[51] H. Bahl, P. Bechtle, S. Heinemeyer, J. Katzy, T. Klingl, K. Peters, M. Saimpert, T. Stefaniak, and G. Weiglein, Indirect $\mathcal{C P}$ probes of the Higgs-top-quark interaction: Current LHC constraints and future opportunities, J. High Energy Phys. 11 (2020) 127.

[52] J. Ellis, D. S. Hwang, K. Sakurai, and M. Takeuchi, Disentangling Higgs-top couplings in associated production, J. High Energy Phys. 04 (2014) 004.

[53] F. Demartin, F. Maltoni, K. Mawatari, B. Page, and M. Zaro, Higgs characterisation at NLO in QCD: $C P$ properties of the top-quark Yukawa interaction, Eur. Phys. J. C 74, 3065 (2014).

[54] M. R. Buckley and D. Goncalves, Constraining the strength and $C P$ structure of dark production at the LHC: The associated top-pair channel, Phys. Rev. D 93, 034003 (2016).

[55] F. Demartin, F. Maltoni, K. Mawatari, and M. Zaro, Higgs production in association with a single top quark at the LHC, Eur. Phys. J. C 75, 267 (2015).

[56] F. Demartin, B. Maier, F. Maltoni, K. Mawatari, and M. Zaro, tWH associated production at the LHC, Eur. Phys. J. C 77, 34 (2017).

[57] A. Kobakhidze, N. Liu, L. Wu, and J. Yue, Implications of $\mathrm{CP}$-violating top-Higgs couplings at LHC and Higgs factories, Phys. Rev. D 95 (2017) 015016.

[58] D. Azevedo, A. Onofre, F. Filthaut, and R. Gonçalo, CP tests of Higgs couplings in $t \bar{t} h$ semileptonic events at the LHC, Phys. Rev. D 98, 033004 (2018).

[59] V. Barger, K. Hagiwara, and Y.-J. Zheng, Probing the Higgs Yukawa coupling to the top quark at the LHC via single top + Higgs production, Phys. Rev. D 99, 031701 (2019).

[60] M. Kraus, T. Martini, S. Peitzsch, and P. Uwer, Exploring BSM Higgs couplings in single top-quark production, arXiv:1908.09100.

[61] D. A. Faroughy, J. F. Kamenik, N. Košnik, and A. Smolkovič, Probing the $C P$ nature of the top quark Yukawa at hadron colliders, J. High Energy Phys. 02 (2020) 085.
[62] B. Bortolato, J. F. Kamenik, N. Košnik, and A. Smolkovič, Optimized probes of $C P$-odd effects in the $t \bar{t} h$ process at hadron colliders, Nucl. Phys. B964, 115328 (2021).

[63] J. Brod, U. Haisch, and J. Zupan, Constraints on $C P$-violating Higgs couplings to the third generation, J. High Energy Phys. 11 (2013) 180.

[64] Y. T. Chien, V. Cirigliano, W. Dekens, J. de Vries, and E. Mereghetti, Direct and indirect constraints on $C P$-violating Higgs-quark and Higgs-gluon interactions, J. High Energy Phys. 02 (2016) 011.

[65] V. Cirigliano, W. Dekens, J. de Vries, and E. Mereghetti, Constraining the top-Higgs sector of the standard model effective field theory, Phys. Rev. D 94, 034031 (2016).

[66] G. Panico, A. Pomarol, and M. Riembau, EFT approach to the electron electric dipole moment at the two-loop level, J. High Energy Phys. 04 (2019) 090.

[67] E. Fuchs, M. Losada, Y. Nir, and Y. Viernik, $C P$ violation from $\tau, t$ and $b$ dimension-6 Yukawa couplings-interplay of baryogenesis, EDM and Higgs physics, J. High Energy Phys. 05 (2020) 056.

[68] W. Beenakker, A. Denner, W. Hollik, R. Mertig, T. Sack, and D. Wackeroth, Electroweak one loop contributions to top pair production in hadron colliders, Nucl. Phys. B411, 343 (1994).

[69] J. H. Kühn, A. Scharf, and P. Uwer, Electroweak corrections to top-quark pair production in quark-antiquark annihilation, Eur. Phys. J. C 45, 139 (2006).

[70] W. Bernreuther, M. Fuecker, and Z.-G. Si, Weak interaction corrections to hadronic top quark pair production, Phys. Rev. D 74, 113005 (2006).

[71] S. Moretti, M. Nolten, and D. Ross, Weak corrections to gluon-induced top-antitop hadro-production, Phys. Lett. B 639, 513 (2006).

[72] J. H. Kühn, A. Scharf, and P. Uwer, Electroweak effects in top-quark pair production at hadron colliders, Eur. Phys. J. C 51, 37 (2007).

[73] M. Aliev, H. Lacker, U. Langenfeld, S. Moch, P. Uwer, and M. Wiedermann, HATHOR: HAdronic top and heavy quarks crOss section calculatoR, Comput. Phys. Commun. 182, 1034 (2011).

[74] T. Martini and M. Schulze, Electroweak loops as a probe of new physics in $t \bar{t}$ production at the LHC, J. High Energy Phys. 04 (2020) 017.

[75] A. Dedes, W. Materkowska, M. Paraskevas, J. Rosiek, and K. Suxho, Feynman rules for the standard model effective field theory in $\mathrm{R}_{\xi}$-gauges, J. High Energy Phys. 06 (2017) 143.

[76] P. Artoisenet et al., A framework for Higgs characterisation, J. High Energy Phys. 11 (2013) 043.

[77] A. Denner, Techniques for calculation of electroweak radiative corrections at the one loop level and results for W physics at LEP-200, Fortschr. Phys. 41, 307 (1993).

[78] J. Alwall, R. Frederix, S. Frixione, V. Hirschi, F. Maltoni, O. Mattelaer, H.-S. Shao, T. Stelzer, P. Torrielli, and M. Zaro, The automated computation of tree-level and next-toleading order differential cross sections, and their matching to parton shower simulations, J. High Energy Phys. 07 (2014) 079.

[79] T. Sjostrand, S. Mrenna, and P.Z. Skands, A brief introduction to PYTHIA8.1, Comput. Phys. Commun. 178, 852 (2008). 
[80] DELPHES 3 Collaboration, DELPHES3, A modular framework for fast simulation of a generic collider experiment, J. High Energy Phys. 02 (2014) 057.

[81] M. L. Czakon et al., Top quark pair production at complete NLO accuracy with NNLO + NNLL' corrections in QCD, Chin. Phys. C 44, 083104 (2020).

[82] S. Catani, S. Devoto, M. Grazzini, S. Kallweit, and J. Mazzitelli, Top-quark pair production at the LHC: Fully differential QCD predictions at NNLO, J. High Energy Phys. 07 (2019) 100.

[83] N. Kidonakis, NNLL threshold resummation for top-pair and single-top production, Phys. Part. Nucl. 45, 714 (2014).

[84] P. Kant, O. Kind, T. Kintscher, T. Lohse, T. Martini, S. Mölbitz, P. Rieck, and P. Uwer, HatHor for single top-quark production: Updated predictions and uncertainty estimates for single top-quark production in hadronic collisions, Comput. Phys. Commun. 191, 74 (2015).

[85] J. Erdmann, S. Guindon, K. Kroeninger, B. Lemmer, O. Nackenhorst, A. Quadt, and P. Stolte, A likelihoodbased reconstruction algorithm for top-quark pairs and the KLFitter framework, Nucl. Instrum. Methods Phys. Res., Sect. A 748, 18 (2014).

[86] LHC Higgs Cross Section Working Group Collaboration, Handbook of LHC Higgs cross sections: 4. Deciphering the nature of the Higgs sector, arXiv:1610.07922.

[87] J. Neyman and E. S. Pearson, On the problem of the most efficient tests of statistical hypotheses, Phil. Trans. R. Soc. A 231, 289 (1933). 